\title{
Packets Wavelets and Stockwell Transform Analysis of Femoral Doppler Ultrasound Signals
}

\author{
M. Latfaoui ${ }^{1}$, F. Bereksi Reguig ${ }^{2}$ \\ ${ }^{1}$ Department of Electrical Engineering, Faculty of Technology, Tahri Mohamed Bechar University, Algeria \\ ${ }^{2}$ Biomedical Engineering Laboratory, Biomedical Engineering Department, Faculty of Technology, \\ University of Tlemcen, Algeria
}

\begin{tabular}{l} 
Article Info \\
\hline Article history: \\
Received Dec 15, 2017 \\
Revised Jan 14, 2018 \\
Accepted Jul 26, 2018 \\
\hline
\end{tabular}

Keyword:

Continuous wavelet transform

Doppler ultrasound

SBI

Stenosis

S-transform

The wavelet packet transform

\begin{abstract}
Ultrasonic Doppler signals are widely used in the detection of cardiovascular pathologies or the evaluation of the degree of stenosis in the femoral arteries. The presence of stenosis can be indicated by disturbing the blood flow in the femoral arteries, causing spectral broadening of the Doppler signal. To analyze these types of signals and determine stenosis index, a number of time-frequency methods have been developed, such as the short-time Fourier transform, the continuous wavelets transform, the wavelet packet transform, and the $S$-transform
\end{abstract}

Corresponding Author:

M. Latfaoui,

Department of Electrical Engineering,

Faculty of Technology,

Tahri Mohamed Bechar University.

Email: m.latfaoui@gmail.com

\section{INTRODUCTION}

Fourier analysis is a basic tool in signal processing. It is indispensable in many areas of research; unfortunately it has limitations when implemented beyond the strict framework of its definition: the area of stationary finite energy signals. In Fourier analysis, all the temporal aspects become illegible in the spectrum. The study of non-stationary signals therefore requires either an extension of the Fourier Transform (or stationary methods), introducing a temporal aspect, or the development of specific methods.

A first solution, implemented intuitively in the mid-century, corresponds to Fourier analysis sliding window or short time Fourier transform (STFT), which was introduced in 1945 by D. Gabor with the idea of a time-frequency plan where time becomes an additional parameter of frequency [1]. This method shows that a joint exact location in time and frequency is impossible, and introduces the idea of a discrete basis, minimum, resulting in a few coefficients of the signal energy distribution in time-frequency plan.

Other methods are used in this work, namely the continuous wavelet transform and wavelet packets. these two variances of the wavelet transform have existed in a latent state in both mathematics and signal processing, but the real expansion began in the early 1980s. The last method used in this work (based on the wavelet transform) is the $S$-trasform proposed by Stokwellet al.; it is similar to STFT with an exception that the amplitude and width of the analysis window are a function of frequency, as in the wavelet transform [2]. 


\section{METHODS OF ANALYSIS}

\subsection{Short-Time Fourier Transform}

This method is based on the decomposition of the signal into small segments in which the Fourier transform is applied; there by generating a localized spectrum analytically STFT is given by the following relationship:

$$
X_{\tau}(f)=\int_{-T / 2}^{T / 2} X(t) w(t-\tau) e^{-j 2 \pi f t} d t
$$

Where $w(t-\tau)$ is a selected window function. The action of this window is to locate in time, the resulting local spectrum. This localization window is then shifted in time to produce the local spectrum for the duration of the existence of $x(t)$. The resulting spectral power is called spectrogram [3]-[5].

\subsection{Continuous Wavelet Transform}

The continuous wavelet transform (CWT) is defined by:

$$
C W T(a, b)=\int_{-\infty}^{+\infty} x(t) \Psi_{a, b}^{*}(t) d t
$$

Where $x(t)$ represents the analyzed signal, $a$ and $b$ represent respectively the scaling factor (dilatation/compression coefficient) and the time (shifting coefficient), and the superscript asterisk $\left(^{*}\right)$ denotes the complex conjugation. $\Psi_{a, b}(t)$ is obtained by scaling the wavelet at time $b$ and scale $a$ :

$$
\Psi_{a, b}(t)=\frac{1}{\sqrt{|a|}} \Psi\left(\frac{t-b}{a}\right)
$$

Where $\psi(t)$ represents the wavelet time function [6].

\subsection{The Wavelet Packet Transform}

Wavelet packets used to decompose the signal to a large number of bases and selected with a certain criterion; the one that best represents the signal. During decomposition, the low-pass and high-pass filtered versions of the signal are decomposed. The approximation of the details and the details of the details are therefore added to the approximation of the signal. The coefficients from this decomposition are characterized by three parameters: the level of decomposition, frequency index, and time index. Each wavelet packet is carrying triple information $\{f, s, p\}$, frequency, scale, and position where the wavelet has only two parameters: scale and position [7]-[8].

In wavelet packet analysis, the signal is decompose into approximations and details. The approximation is then itself decomposed into approximation and detail in second level, and the process is repeated. For a decomposition of $n$ level, there are $(n+1)$ possibilities to decompose or to code the signal. The Figure 1 shows the decomposition of a digital signal in wavelet transform at three levels.

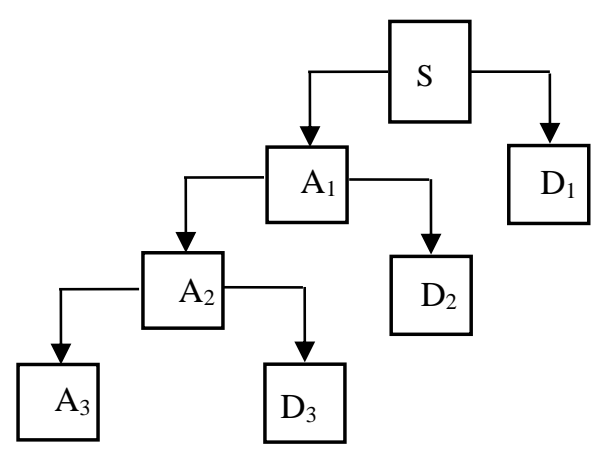

Figure 1. Wavelet transform decomposition scheme 
Where $S=A_{1}+D_{1}, S=A_{2}+D_{2}+D_{1}, S=A_{3}+D_{3}+D_{2}+D_{1}$. In wavelet packets analysis, decomposition into approximation and detail is made only on approximations but also on details. In other words, when analyzing in wavelet packets, it is no longer only the filtered low-pass versions of the signal that are decomposed, but also the filtered high-pass versions. In another way, the high frequencies are also cut into sub-bands and the decomposition tree deviate symmetrically. The wavelet packets decomposition leads to a decomposition into frequency sub-bands of the signal [9]-[11].

\subsection{The S-Transform}

The $S$-transform provides a time-frequency representation of a signal. It only combines a dependent frequency resolution with simultaneous location of the real and imaginary part of the spectrum. It was published for the first time in 1996 by Stokwell. The basic idea of this time-frequency distribution is similar to the Fourier transform sliding window, except that the amplitude and width of the analysis window are variable depending on the frequency as is the case in wavelet analysis [11]-[12]. The $S$-transform of a function $x(t)$ can be defined as a transform into wavelets with a quite specific mother wavelet multiplied by a phase factor:

$$
S(\tau, f)=e^{i 2 \pi f \tau} W(\tau, d)
$$

Where $W(\tau, d)$ is the continuous wavelet transform of the signal $x(t)$ defined by:

$$
W(\tau, d)=\int_{-\infty}^{+\infty} x(t) w(t-\tau, d) d t
$$

Where the mother wavelet is defined by:

$$
w(t, f)=\frac{|f|}{\sqrt{2 \pi}} e^{-\frac{t^{2} f^{2}}{2} e^{-i 2 \pi f t}}
$$

Let us note that the dilatation factor $d$ is the reverse of the frequency $f$ [13]-[14].

\section{RESULTS AND ANALYSIS}

The Doppler signals studied in this work are from the $S^{t}$ Marie hospital in Leicester (England); the signal files are in .wav form with a sampling frequency of $44 \mathrm{KHz}$ and a duration of $4.34 \mathrm{~s}$, corresponding to 191390 samples. The Figure 2 shows a temporal representation and spectral analysis of a signal database. From the results of Keeton and Sadik given in [15]-[16], a Doppler signal can be regarded as a Gaussian signal in a segment of $10 \mathrm{~ms}$ to $12 \mathrm{~ms}$. Although it has not always guaranteed that the Doppler signals are Gaussian at $10 \mathrm{~ms}$, this remains true for segments below $10 \mathrm{~ms}$.

The principal objective of this research is to compare STFT, CWT, PWT and S-transform methods in the case of the resolution of time-frequency of ultrasonic Doppler signals. The purpose of the timefrequency analysis is to provide a more informative description of the signal revealing the temporal variation of its frequency content. A solution, which is considered as the more intuitive, consists in associating a nonstationary signal a sequence of Fourier transforms short-term to adapt the successive observation windows to structural variations of the signal in such a way that the stationarity assumptions are locally satisfied [16]. The disadvantage of the Fourier transform is the stationary of signals, and therefore does not allow obtaining time information. STFT implicitly is considered for a non-stationary signal as a series of quasi-stationary situations across the analysis window.

The temporal resolution of such an analysis is determined by the width of the window, the frequency resolution is determined by the width of its Fourier transform. For a highly non-stationary signal as an ultrasonic Doppler signal, good temporal resolution is required, which requires working with a short window. The major disadvantage of this transform is the limitation of the frequency resolution. The problem of the STFT is that it uses a fixed size of window covering the time-frequency domain. Another disadvantage of this transform is the fixed temporal and frequency resolution [17]. Processing in general, or by using wavelet S-transform, offer the possibility to have a window that adapts according to the irregularities of the 
signal. Wavelets are a family of functions localized in time and frequency and form an orthonormal basis. They are generated one from the other by translation and dilatation. Each wavelet is used to decompose the signal and is used as each exponential function in the Fourier transform. The difference is that the wavelet functions are well localized in time unlike exponentials [18].

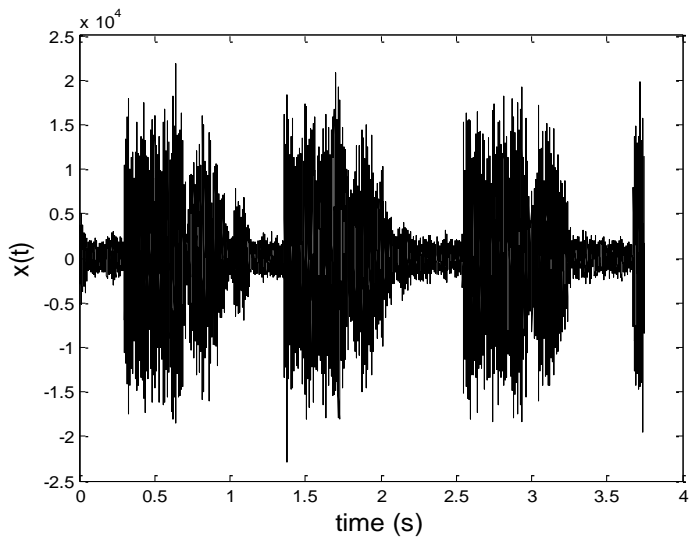

(a)

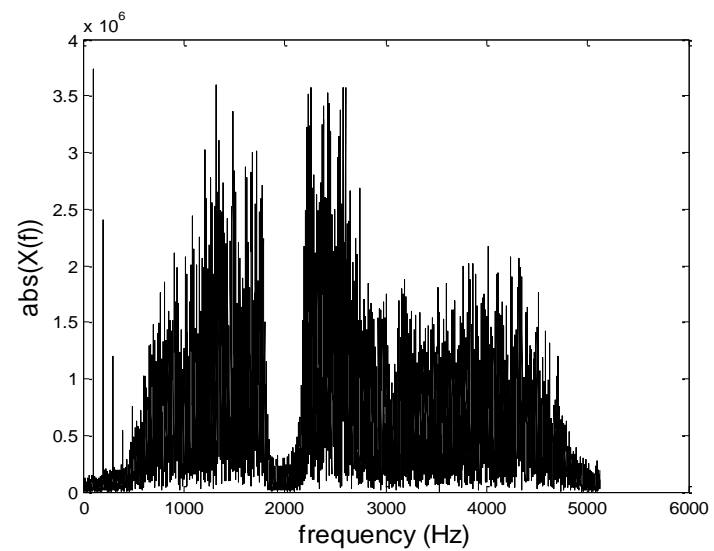

(b)

Figure 2. (a) Temporal representation. (b) Spectrum of Doppler Ultrasound Signal

The wavelet packets transform is a generalization of the DWT; it allows decomposing the details approximation. This transform allows decomposing the Doppler signal into sub-frequency bands by means of a filter bank. The coefficients of this decomposition give a time-frequency representation that can monitor the velocity of blood in the arteries. The wavelet-based transform like CWT, PWT or S-transform are designed to give good time resolution with a poor frequency resolution at high frequencies and a good frequency resolution with a poor temporal resolution at low frequencies. The major drawback of these transforms is the choice of the mother wavelet. Using the STFT or wavelet-based transform requires a compromise between time and frequency resolutions. For the STFT, narrower analysis window will provide better temporal resolution, but the concentration around the origin of the Fourier transform will necessarily be less, which implies a poorer frequency resolution. For further transforms, the compromise is similar, and depends on the scan frequency: increasing the analysis frequency implies improving the time resolution, but decreasing the frequency resolution [15].

The wavelet-based transforms have been designed for non-stationary signals since they incorporate the concept of scale to transformation, which gives better time-frequency resolution: a compressed wavelet to analyze the high frequency detail and a dilated wavelet to detect underlying trends of low frequency. In sonograms obtained by STFT, CWT, PWT and S-transform are given in Figure 3. The horizontal axis $(t)$ shows the time and the frequency $(f)$ is shown on the vertical axis. The gray level intensity represents the power level corresponding to a frequency for each point in the time axis [19].

It is clear that the $C W T, P W T$ and $S$-transforms could help to improve the quality of sonograms of Doppler. Ultrasonic Doppler signals sampled contain a wealth of information on blood flow. The most comprehensive way to show this information is to perform a time-frequency analysis and present the results as sonograms. For the $S$-transform, a linear time-frequency representation is presented. This method surpasses the problem of the sliding window Fourier transform of fixed length, and addresses the notion of phase in the wavelet transform for non-stationary signals analysis. This transform provides a very suitable space for feature extraction and location in time and frequency discriminating information in the ultrasonic Doppler signal [14].

In Figure 3, one can observe an improvement in quality of sonograms obtained by the wavelet transform over those obtained by STFT. The sonograms obtained by STFT give a low-quality spectral interpretation in terms of location of minimum and maximum frequencies. The advantage of the $S$-transform is optimizing the time-frequency resolution.

It is clear from Figure 3 that there is a certain qualitative improvement in sonograms obtained by the $S$-transform compared to those obtained by STFT. Sonograms obtained by STFT give false frequencies, and spectral analysis by the STFT produces unclear sonograms because of the distortionin spectral estimation caused by sliding window. The advantage of the $S$-transform compared to STFT is the optimization of timefrequency resolution and the dynamic localization of the spectrum in the time-frequency plan. The second

Packets Wavelets and Stockwell Transform Analysis of Femoral Doppler Ultrasound Signals (M. Latfaoui) 
advantage of the $S$-transform is a better location of systolic peaks used to determine the spectral broadening index $(S B I)$.

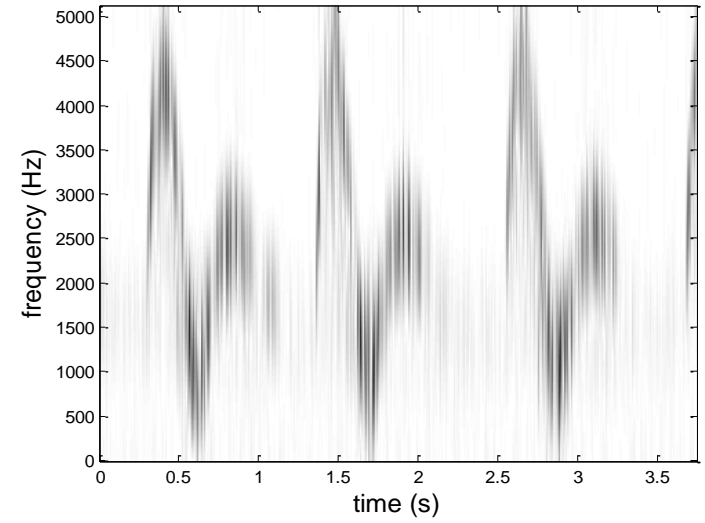

(a)

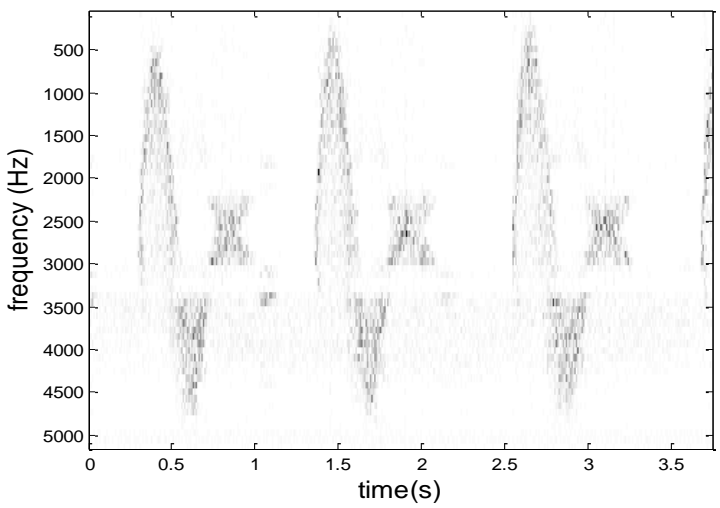

(c)

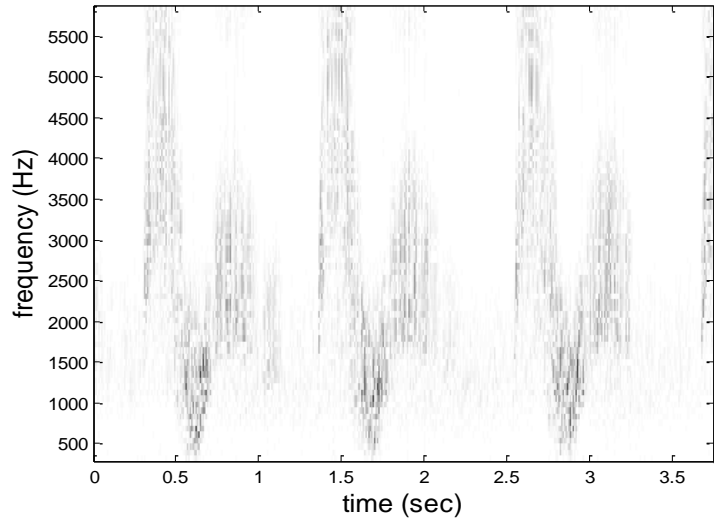

(b)

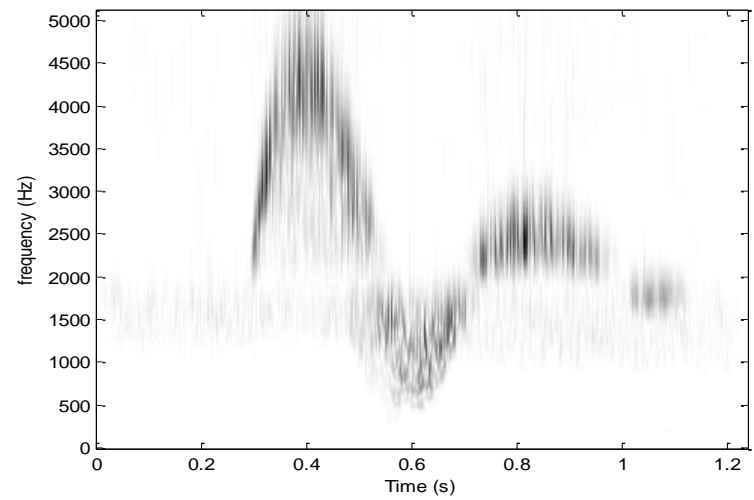

(d)

Figure 3. Femoral arterial Doppler sonograms: (a) using STFT, (b) using CWT, (c) using $P W T$, (d) using $S$-transform

Particularly because of the intrinsic limitations of the spectrogram and particularly time-frequency resolution of problems, other types of frequency-time representation are preferred. The time-frequency and time-scale analysis's have been developed to meet a need for demonstrating phenomena that are very localized in time and frequency. Unlike the spectrogram, the resolution of the time-frequency representation obtained by the $S$-transform or wavelet-transform is dependent on frequency and time. Both well localized in time and frequency, the S-transform or wavelet transform has properties of "zoom" making it an ideal tool for detecting phenomena of high frequency and short duration. It is worth reminding that wavelet transforms possess a good temporal resolution at high frequencies and the vice-versa [19]. The wavelet transforms utilize a set of analytical functions built by expansion / compression and translation of a function called mother wavelet. In our study, we chose the Morlet wavelet that has a similar form to Doppler ultrasound signals [20].

\section{CALCULATING SBI}

Speeds of the red blood cells in a vessel are determined from the Ultrasound Doppler signals. The temporal evolution of these echoes is sonograms. A variation of these speeds translates directly a variation frequency, or temporal level sonogram. In fact, such situations exist where structures are found in the arteries (carotid or femoral). These directly affect the blood flow that becomes non-uniform in their neighborhood. This causes enlargement of the Doppler signal spectrum near the systolic peak quantified by what we call the 
spectral broadening index $(S B I)$. However, this index is highly correlated with the nature of the envelope frequency $\left(f_{\max }\right.$ and $\left.f_{\text {mean }}\right)$. In fact, the frequency generated from sonograms is embedded in noise emanating from different sources reflecting (the wall of the artery, skin, etc.) that can see in Figure 4.

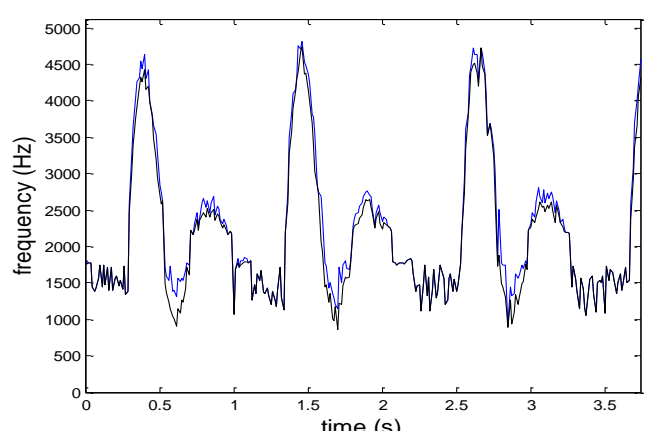

(a)

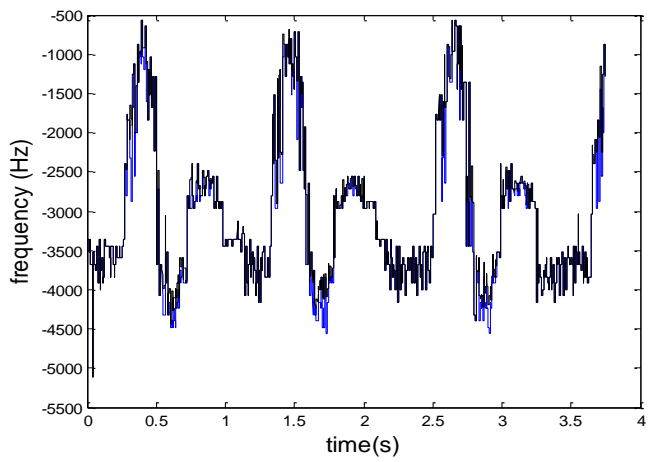

(c)

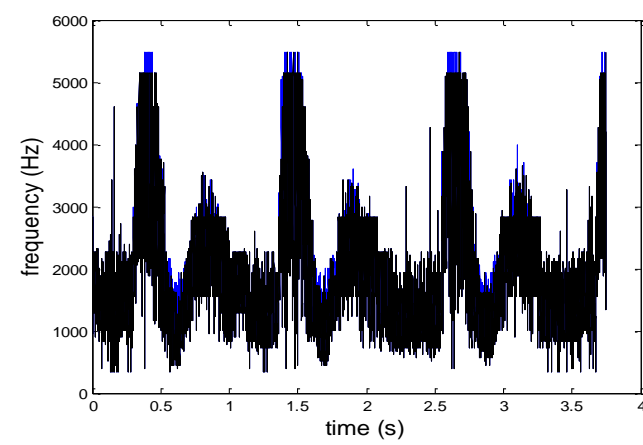

(b)

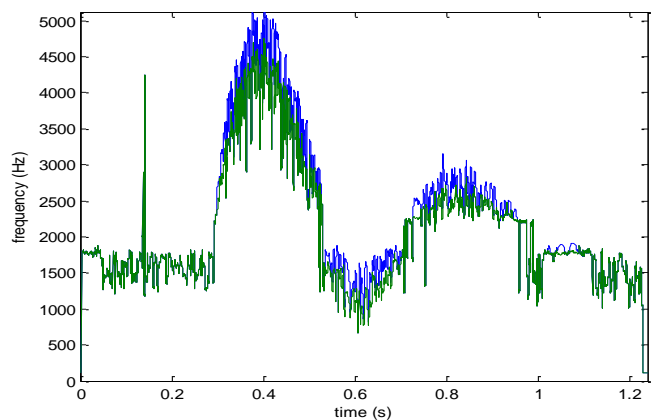

(d)

Figure 4. Maximum and mean frequency envelopes of the femoral arterial extracted from (a) STFT, (b) CWT, (c) PWT, (d) S-transform sonograms

A filter applied to the spectral envelopes is required to determine the systolic peak and estimate the SBI index. The Figure 5 illustrate the systolic peaks that are clearly defined and thus allowing better temporal location of systolic evolution. The severity of the stenosis (given in percentage \%) is expressed by the ratio of the diameter reduced by the stenosis and the actual diameter of the artery.

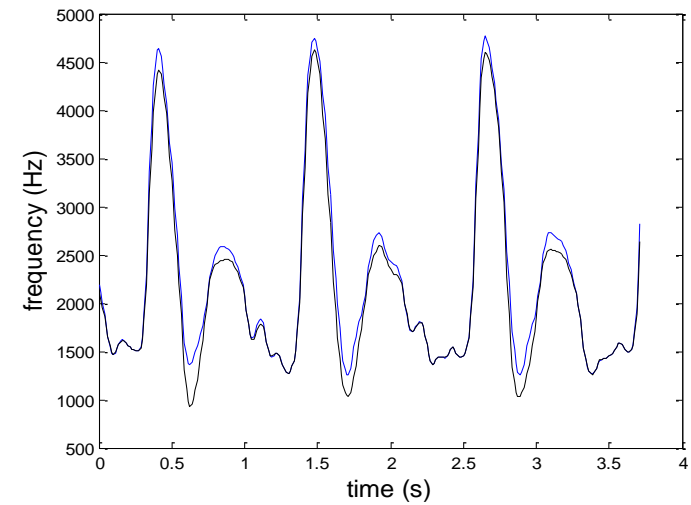

(a)

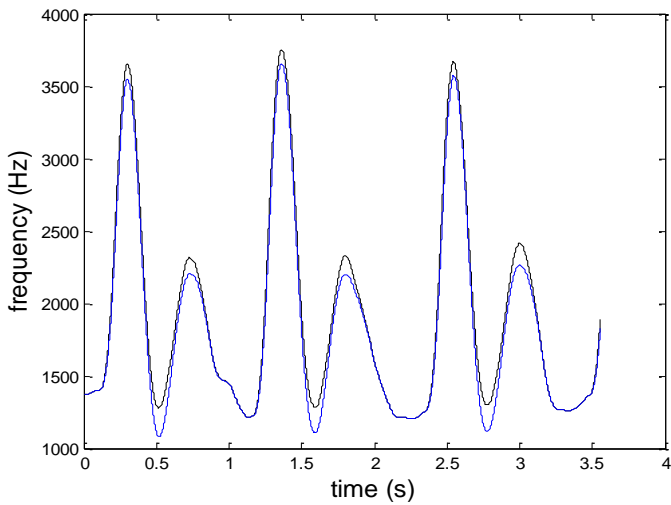

(b)

Figure 5. Filtered envelopes fmax and fmean of the artery femoral snogramms: (a) using STFT, (b) using CWT 


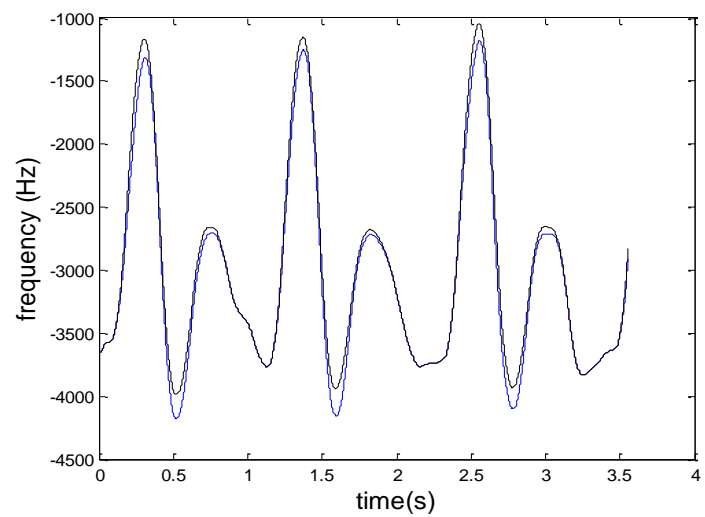

(c)

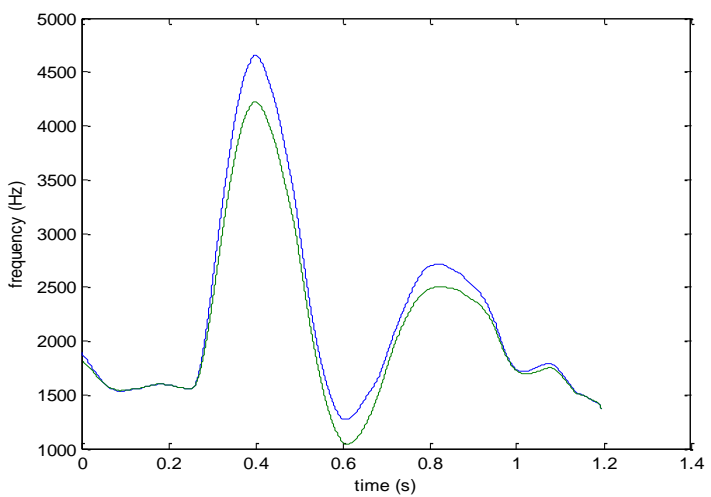

(d)

Figure 5. Filtered envelopes fmax and fmean of the artery femoral snogramms: (c) PWT, (d) using Stransform

In Figure 6, the upstream flow of the stenosis is laminar and red blood cells flow with a speed called average speed $V_{\text {mean }}$. At the stenosis, the red blood cell velocity increases because of arterial constriction, and this maintains a constant flow. In this case, the speed is maximum and is called $V_{\max }$. Immediately down stream of the stenosis, when the diameter increases, suddenly appears vortexed completely disrupting the flow, this allows the red blood cells to take multiple speeds and in all directions. The average value of these speeds gives the average flow velocity $V_{\text {mean }}$ [15]. Since the speed $V_{\max }$ and $V_{\text {mean }}$ respectively represent the frequencies $f_{\max }$ and $f_{\text {mean }}$ Doppler spectrum, one can express the degree of stenosis according to $f_{\text {max }}$ and $f_{\text {mean }}$ :

$$
S B I=\frac{f_{\max }-f_{\text {mean }}}{f_{\max }}
$$

According to this equation, the $S B I$ is then used in our study to calculate the degree of stenosis. Since the $S B I$ is calculated by the ratio of $\left(f_{\max }-f_{\text {mean }}\right)$ and $f_{\max }$, it is necessary to use larger values of $f_{\max }$ and $f_{\text {mean }}$ by the fact that using small values may introduce a important errors in the $S B I$ calculation. For this reason, the $S B I$ is calculated at the systolic peak, where the flow rates (frequencies of the Doppler spectrum) are maximal. The degree of severity of the stenosis, (given in percentage \%) is expressed by the diameter reduced by the stenosis and the real artery diameter [15].

$$
\text { Degree of stenosis }=\frac{(A-B)}{A} \times 100 \%=\frac{V_{\max }-V_{\text {mean }}}{V_{\max }} \times 100 \%
$$

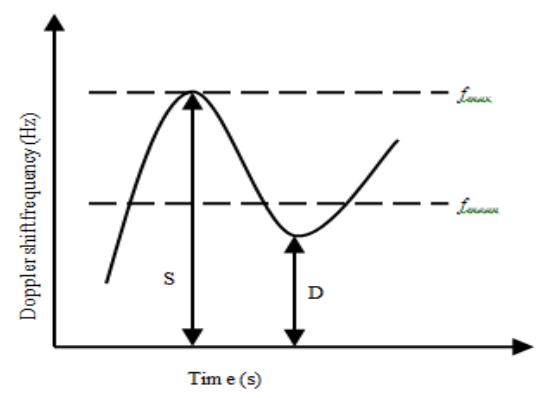

(a)

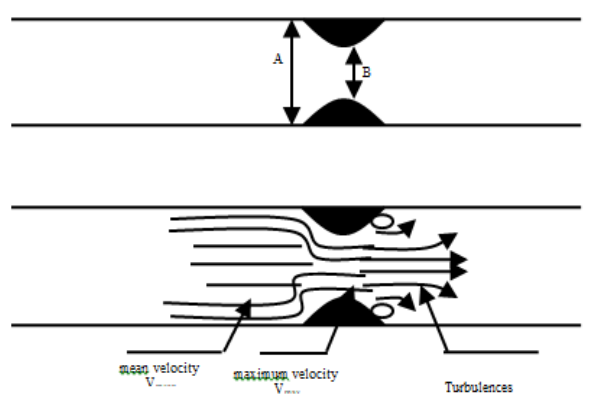

(b)

Figure 6. (a) Diagram illustrating the variable involved in the definition of SBI. $f_{\max }$ is the maximum frequency at peak systole, $f_{\text {mean }}$ is the mean frequency, $\mathrm{S}$ is systolic peak, $\mathrm{D}$ is end diastolic height.

(b) the effect of stenosis on the flow of blood in the arteries 
There are two methods of calculation: The first method is to calculate the average parameters $\left(f_{\max }\right.$ and $f_{\text {mean }}$ ) of each systolic peak, and then to deduce the $S B I$. The second method is to average the $S B I$ found from the parameters $\left(f_{\max }\right.$ and $\left.f_{\text {mean }}\right)$ of each systolic peak. The $S B I$ different values calculated by various methods applied on STFT, $S$-transform, $C W T$ and $P W T$ modeling transform techniques are illustrated on the Figure 7.

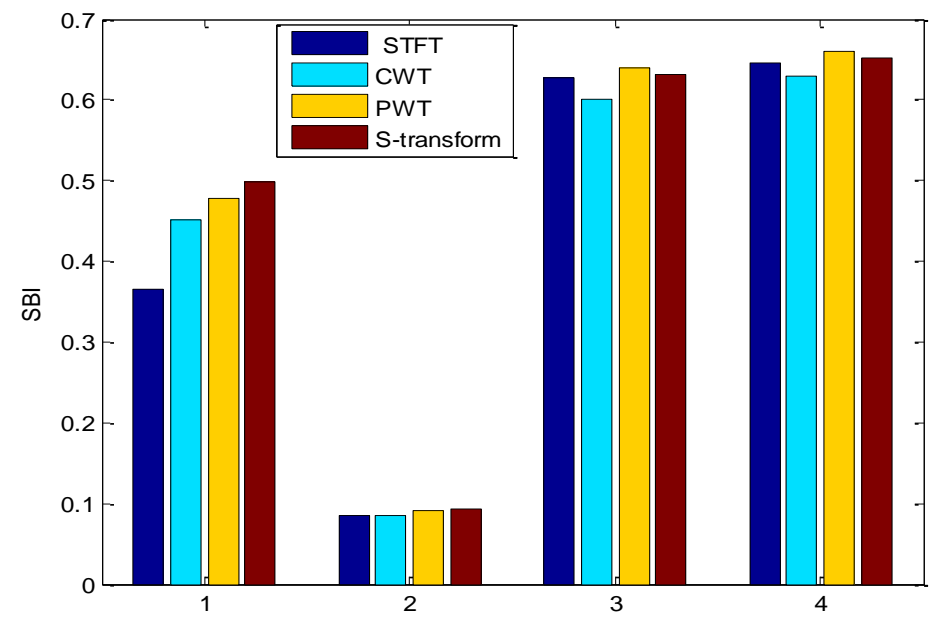

Figure 7. SBI magnitude of differents Femoral Doppler signals using: STFT, CWT, PWT and $S$-transform methods

These results show that the measurement of spectral broadening quantified by broadening spectral index may be an indication of stenosis severity at the femoral arteries. The $S B I$ was calculated from the STFT, $S$-transform, $C W T$ and $P W T$ sonograms. One observed a strong correlation between the value of the SBI obtained by STFT and that obtained by $S$-transform, $C W T$ and $P W T$. The results of this study proved that in spite of the qualitative improvement of the different sonograms; it has no quantitative advantage in employing the S-transform, $C W T$ and PWT compared to the STFT for the determination of SBI due to its weak variance and with the additional numerical requirements.

\section{CONCLUSION}

The time-frequency analysis methods used in this work aim to show the sonograms of ultrasonic Doppler signals, wavelet-based methods such as CWT, PWT and S-transfrom and the classical STFT method have been compared in terms of their frequency-resolving power and their effects in determining the spectral broadening index in the presence of the stenosis in the ultrasound Doppler signals of the femoral arteries.

\section{REFERENCES}

[1] M. Jianping and J. Jin, "Analysis and design of modified window shapes for S-transform to improve timefrequency localization," Mechanical Systems and Signal Processing, vol. 58, pp. 271-284, 2015.

[2] S. Kara, "Classification of mitral stenosis from Doppler signals using short time Fourier transform and artificial neural networls," Expert Systems witn applications, pp. 468-475, 2007.

[3] S. Dhanyar and V.S. K. Roshni, "Comparison of various texture classification methods using multiresolution analysis and linear regression modelling," SpringerPlus, 2016.

[4] F. Dirgenali, et al., "Estimation of wavelet and short-time Fourier transform sonograms of normal and diabetic subjects' electrogastrogram," Computers in Biology and Medicine, vol. 36, pp. 1289-1302, 2006.

[5] J. S. Ashwin and N. Manoharan, "Audio Denoising Based on Short Time Fourier Transform," Indonesian Journal of Electrical Engineering and Computer Science, vol. 9, pp. 89-92, 2018.

[6] V. Thiyagarajan and N. P. Subramaniam, "Analysis and Estimation of Harmonics Using Wavelet Technique," TELKOMNIKA Indonesian Journal of Electrical Engineering, vol/issue: 13(2), pp. 305-313, 2015.

[7] K. Satyanarayana, et al, "Identification of Faults in HVDC System using Wavelet Analysis," International Journal of Electrical and Computer Engineering, vol. 2, pp. 175-182, 2012.

[8] M. Vatani, "Transient Analysis of Switching the Distributed Generation Units in Distribution Networks," International Journal of Applied Power Engineering, vol. 5, pp. 130-136, 2016. 
[9] P. Soundiraraju and N. Loganathan, "Wavelet Transforms Based Power TransformerProtection from Magnetic Inrush Current," TELKOMNIKA Indonesian Journal of Electrical Engineering, vol. 14, pp. 381-387, 2015.

[10] Z. Guo, "Time-frequency representation and pattern recognition of Doppler Blood Flow Signal for Stenosis Classification," PHD thesis, McGill University, Montreal, 1993.

[11] G. Serbes, et al., "Directional dual-tree complex wavelet packet transforms for processing quadrature signals," Medical \& Bio.Eng. \& Computing, vol. 54, pp. 295-313, 2014.

[12] Z. Zhang, et al., "Time Frequency Wavenumber Analysis of Surface Waves and Signal Enhancement Using Stransform," J. Comp. Acous, vol. 23, 2015.

[13] Z. Bouguila, et al., "A new optimized Stockwell transform applied on synthetic and real non-stationary signals," Digital Signal Processing, vol. 46, pp. 226-238, 2015.

[14] H. K. Vydana and A. K. Vuppala, "Detection of fricatives using S-transform," The Journal of the Acoustical Society of America, vol/issue: 140(5), pp. 3895-3907, 2016.

[15] S. Kara, et al., "Spectral broadening of lower extremity venous Doppler signals using STFT and AR modelling," Digital Signal Processing, vol. 18, pp. 669-676, 2008.

[16] P. I. J. Keeton and F. S. Schlindwein, "Spectral broadening of clinical Doppler signals using FFT and autoregressive modelling," European Journal of Ultrasound, vol. 7, pp. 209-218, 1998.

[17] F. S. Schlindwein and D. H. Evans, "Selection of order of autoregressive model for spectral analysis of Doppler ultrasound signals," Ultrasound Med Biol, vol/issue: 16(1), pp. 81-91, 1990

[18] Y. Zhang, et al., "Correction for broadening in Doppler blood flow spectrum estimated using wavelet transform," Medical Engineering \& Physics, vol. 28, pp. 596-603, 2006.

[19] X. S. Li, et al., "Analysis and Simplification of Three-Dimensional Space Vector PWM for Three-Phase Four-Leg Inverters," IEEE Transactions on Industrial Electronics, vol. 58, pp. 450-464, 2011.

[20] K. Kaluzynski and T. Palko, "Effect of method and parameters of spectral analysis on selected indices of simulated Doppler spectra," Med. Biol. Eng. Comput., vol. 31, pp. 249-256, 1993. 